\title{
SURF Points Versus SIFT Points in Identification of Medicinal Plants
}

\author{
PL. Chithra, S. Janes Pushparani
}

\begin{abstract}
Today, digital image processing is used in diverse fields; this paper attempts to compare the outcome of two commonly used techniques namely Speeded Up Robust Feature (SURF) points and Scale Invariant Feature Transform (SIFT) points in image processing operations. This study focuses on leaf veins for identification of plants. An algorithm sequence has been utilized for the purpose of recognition of leaves. SURF and SIFT extractions are applied to define and distinguish the limited structures of the documented vein image of the leaf separately and Support Vector Machine (SVM) is integrated to classify and identify the correct plant. The results prove that the SURF algorithm is the fastest and an efficient one. The results of the study can be extrapolated to authenticate medicinal plants which is the starting step to standardize herbs and carryout research.
\end{abstract}

Keywords: digital image processing, foliage, herbal, medicinal plants, leaf vein, Scale Invariant Feature Transform (SIFT) points extraction, Speeded Up Robust Feature (SURF) points extraction, Support Vector Machine (SVM) classifier.

\section{INTRODUCTION}

Plants are beyond doubt useful for the protection of the environment. However, it is a complicated and mandatory assignment to recognize the varieties of plants in the land. The perilous situation is that several plants are notified as rare and endangered species.

Based on the theory of plant classification, plants are primarily categorized depending on the shape of flowers, fruits and leaves. Identification of leaf contributes to a vivacious role in classification of plants. Therefore, in contrivance using the leaf and leaf veins, the identification features of the plants are extracted and the mined features are fed-in as basic data for the classifiers to categorize the plants. Each image of a leaf is categorized by a series of interrelated procedures. Primarily, a catalogue was initiated by means of model imageries for each variety of plants. Individual pictograph of leaf is associated with the equivalent particulars of the herbs. After the pictograph of leaf is transferred to the personal computer, its indispensable structures are recognized as well as documented by means of image processing methods.

Revised Manuscript Received on December 30, 2019.

* Correspondence Author

PL. Chithra*, Professor Department of Computer Science, University of Madras,Chennai, India. E-mail: chitrasp2001@yahoo.com

S. Janes Pushparani, Assistant Professor, Department of Computer Applications, Ethiraj College for Women, Chennai, India. E-mail: janes_pr@yahoo.com

(C) The Authors. Published by Blue Eyes Intelligence Engineering and Sciences Publication (BEIESP). This is an open access article under the CC BY-NC-ND license (http://creativecommons.org/licenses/by-nc-nd/4.0/)
This study concentrates by recognizing the title of the herbs by analyzing the foliage vein. Recognition will be determined on the basis of limited catalogue. Each stint a foliage is recorded, it shall inevitably generate a file christened in the name of the herbs. The RGB imageries are altogether handled and sifted to obtain its foliage veins. Nevertheless, if the name of the herb is not found in the catalogue, the individual can apprise it later after the herbs are recognized. This project focuses on the variety of foliage pertaining to 7 diverse types of herbs through 10 repetitions for each herb which was established for herbal documentation.

Digital image processing techniques have been utilized to automate identification of plant species through its leaf veins. Different steps involved in leaf recognition are:

$>$ Image acquisition.

$>$ Image pre-processing (noise removal, resize)

$>$ Feature extractions

$>$ Classification

$>$ Identification/recognition

This manuscript is systematized in the following manner. Section II explains the associated methodologies in this field, Section III depicts the methodologies to obtain the feature extractions of leaf vein. Section IV presents the observation of the experiments. Section $\mathrm{V}$ analyses the results and finally Section VI yields the conclusion.

\section{RELATED WORK}

Several investigators have put forth concerted efforts to identify and authenticate varieties of herbal species. Plants so far have been identified based on its texture information, plant image, color histogram, and edge features. By using neural networks, plants have been classified as herbs, shrubs and trees.

The approach by Sandeep Kumar.E [1] is based on leaf color, leaf area and edge features. From obtaining the variance in frame, color histogram and edge histogram, the correct leaf image is identified. The pattern recognition for different kinds of medicinal leaves data was realized and then recognized by means of Canny detector and neural network algorithm in C. Ananthi [2] to identify the leaf image. Jesse Dave S. Selda [3] uses the plant biometrics such as leaf veins and leaf contour characteristics by using Scale Invariant Feature Transform (SIFT) points and Support Vector Machine (SVM) and implementing a raspberry $\mathrm{Pi}$, a low priced, rudimentary inherent structure using a single board computer to recognize the leaf image. Vijayashree .T and A. Gopal [4] experimented sorting of herbs using features of leaf namely identical contour, venation, consistency and color and parameters like aspect ratio, compactness, centroid, eccentricity and dispersion. 
Sachin D. Chothe and V. R. Ratnaparkhe [5] projected a method to recognize herbs depending on the following parameters: form factor, aspect ratio, rectangularity, narrow factor, perimeter ratio of diameter and perimeter ratio of length and width. Pushpa BR [6] described a methodology by using parameters such as convex hull ratio, isoperimetric quotient, eccentricity and entropy. Leaf factors for different leaf images of each species are separately calculated and stored in the database. The system finds the leaf factors for the new image and matches with the database. James S. Copq. [7] explained an approach by analyzing leaf shape, venation extraction, leaf margin, leaf texture, lamina-based methods, flower and other plant organs. Kue - Bem Lee [10] presented a method by means of leaf vein extraction and leaf feature extraction. Using this approach main vein is extracted for the pointer pictograph and foliage track is resolute by means of forecast histogram of mined core vein pictograph and produced a leaf recognition system.

Considering the above facts, a modest attempt has been put-forth by taking into account the morphological particulars of the herb deprived of numerous complications. Various scholars have tried out various methodologies for eliciting the parameters. But this effort customizes a modest and vigorous method by means of Speeded Up Robust Feature (SURF) points. Amongst the different edge detection procedures, Canny edge detection algorithm has been utilized that abstracts the borderline pattern including the vein pattern effectively.

\section{METHODOLOGY}

Fig. 1 demonstrates the over-all background of foliage documentation structure. The old-style phases are utilized so as to recognize a herb, if a pictograph of a foliage is provided as hereunder.

With respect to herbal foliage cataloguing, the primary phase is digitization. Foliage pictograph is acquired using digital camera and that image is labelled as an input pictograph.

The input pictograph is preliminary processed to augment the significant topographies. Revamping procedure comprises pictures that are to be transformed into monochrome, pictograph separation i.e the process of segregating a binary pictograph to digital adaptation,

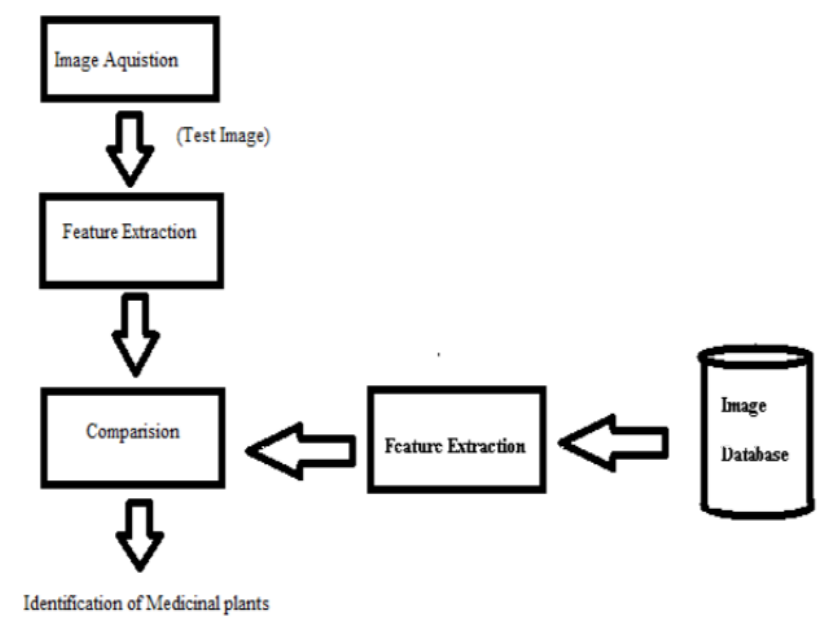

Fig. 1. System block diagram [1]

pictograph levelling and manifold sections. The aim of the preliminary processing of pictograph is to display symbols of enhancement in receiving pictograph info. This will help to overcome undesirable data and it focusses on magnifying the appropriate pictograph topographies for supplementary processing.

The succeeding procedure is that the important attribute of leaf vein is drawn and the SURF points and SIFT points are identified. The input pictograph is catalogued to the herb pertaining to the foliage pictograph encompasses maximum outlier matching score by means of SVM, afterwards the data for the input pictograph is attained.

Figure 1 displays the order by which the pictograph input is transformed to yield the designated output nomenclature of herb. The acquired pictograph of foliage vein pattern assists for the input and for the processor to recognize then undergoing the procedure of pattern recognition and feature extraction algorithms.

The recognition of foliage is carried out by image processing algorithm. A phased procedure is demonstrated in Fig. 2. The pictograph of the foliage is acquired using a digital camera beneath a white backdrop for distinguishing the sharp features of the foliage and as well for the improved precision. Pattern recognition consists of pixelization, linear filtering and quantization. The features are extracted which are precise to various foliage species by means of usage of the foliage data. The method of preprocessing of the pictograph comprises the decrease of noise or distortion, enhancement of pictograph for improved interpretation and pictograph sharpening for sensing the shrill boundaries and veins pertaining to leaf. The subsequent and concluding step is execution. The apprehended topographies are mined and distinguished by means of the database and the outcome is attained. The catalogue is to be designed with numerous trials of almost 300 samples of foliage. Additional trials are tried to recognize the points or values to obtain the meticulous coincidence.

Fig. 3 shows the software schema. The aforesaid operation that has to be skilled should intelligently categorize herbs correctly [3]. The input pictograph shall be dealt through an algorithm aimed at feature extraction and pattern categorization. Subsequent to this exercise, it is inferred that the leaf is equipped for documentation.

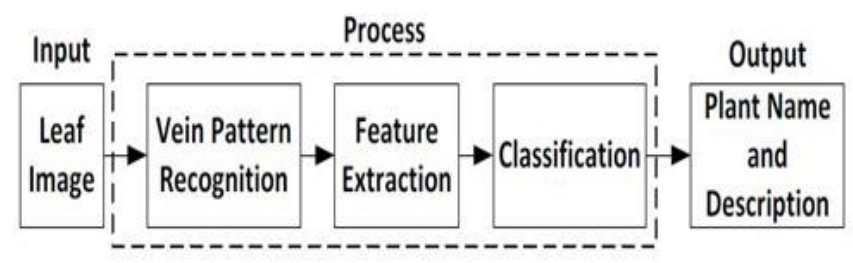

Fig. 2. Diagram for Leaf Recognition

\section{A. SURF Subroutine}

Fig. 4 displays the phases of the image processing of input pictograph. Foliage pattern identification begins on or after colour conversion. SURF could be utilized to define and distinguish the limited 


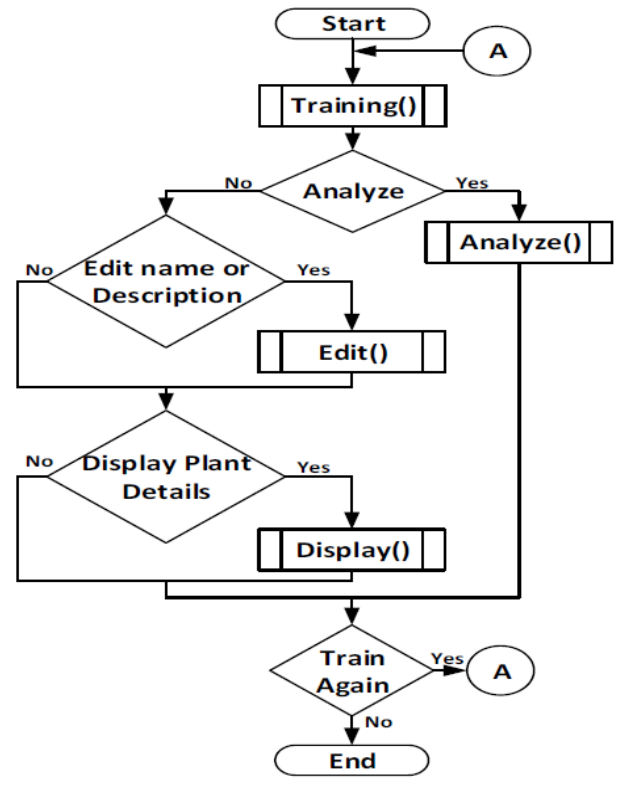

Fig. 3. System Flow chart

topographies of a pictograph. SVM requires a novel input pictograph beginning with the SURF that would be equated to the matrix stored in the database. Then SVM would categorize that possess the maximum notch is said to be exhibited.

SURF (Speeded Up Robust Features) is defined as gauge and revolution invariant interest point detector and descriptor. SURF comes close to LoG by means of Box Filter. The greatest benefit to do this scheming is convolution through box filter through the aid of integral pictographs was possible by means of simple calculation. Moreover, this can be done simultaneously for diverse scales. In addition, SURF

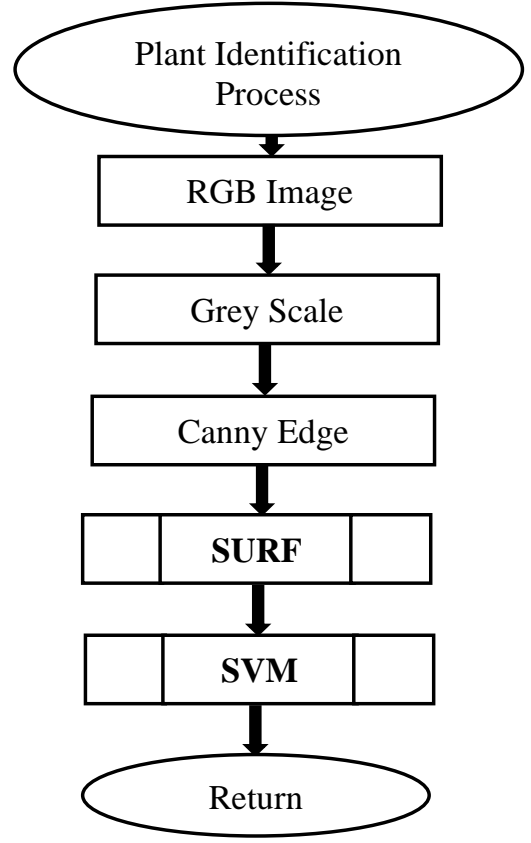
SIFT Subroutine

relies based upon determinant of matrix Hessian aimed at location as well as scale. With regard towards orientation assignment, the aforementioned utilizes wavelet replies for horizontal as well as vertical route by means of utilizing satisfactory Gaussian weightiness. SURF customs the wavelet replies meant for feature portrayal. A zone near the significant point is carefully chosen which was divisible into
Fig. 4 Leaf Recognition Flow chart using SURF

sub sections and then for each sub section the wavelet replies are recorded and characterized to obtain the SURF feature descriptor. The symbol of Laplacian that was previously computed for the detection will be utilized for fundamental and significant points. For the purpose of distinguishing shiny and glowing blobs in dusky background as of the reverse case, the sign of the Laplacian is useful.

Fig. 4 illustrates the procedure for the executing SURF investigation by MAT LAB. SURF points of significance objects would be foremost mined as established pictographs of reference and warehoused in the catalogue which becomes a trained set. Then the new image is recognized individually by comparing each feature with the catalogue so as to discover the exact entrant match feature depending on Euclidean distance of the feature vectors.

Fig. 5 displays the phases of the image processing of input pictograph. Foliage design starts after colour conversion. SIFT would be utilized to define and distinguish the limited topographies of a pictograph. SVM requires a novel input pictograph after the SIFT would be equated to the matrix stored in the catalogue. Thereby SVM would categorize that has the maximum notch and it will be exhibited.

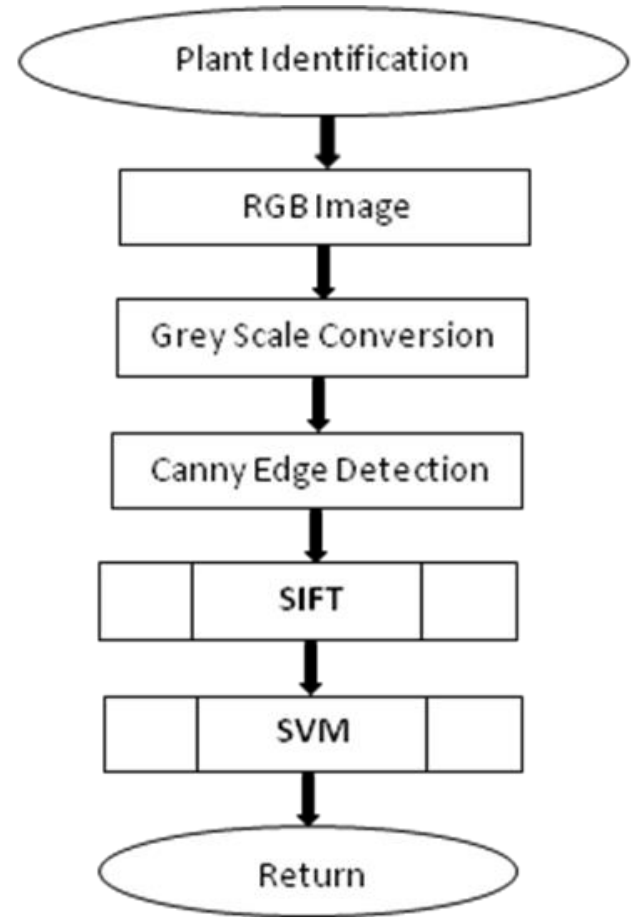

Fig. 5 Leaf Recognition Flow chart using SIFT

SIFT (Scale Invariant Feature Transform) key points were initially mined using a group of resource pictographs and warehoused in a catalogue. In the novel pictograph each feature will be independently compared with the catalogue as well as the entrant will be matched depending upon Euclidean distance pertaining to feature vectors. SIFT topographies depends upon the presence of the entity in precise concentration points that were invariant to pictograph scale and rotation.

\section{B. SVM Subroutine}

Support Vector Machine is a machine learning method utilized to sort as well as regression analysis which is represented in Fig. 6.

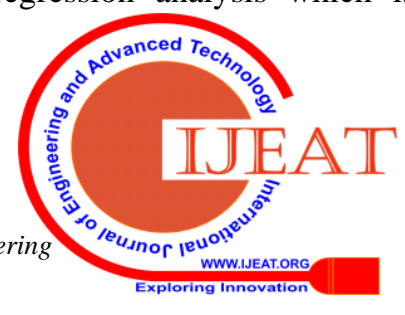


This rests on replicas and becomes proficient through algorithm education. Therefore, huge quantum of data is being evaluated to recognize topographies after it.

The aim of the SVM classifier is to befit the facts obtained from the SURF/SIFT catalogue and then to return the finest match based on the pictograph classes of the facts.

\section{RESULT}

Experiments are made on database. The preliminary phase carried out is to predispose by what the color image is transformed to grayscale as well as how the edges are distinguished using Canny edge detection algorithm for deciding the shrill topographies of the pictograph. Feature extraction stands accomplished by using SURF points that are represented by Fig. 7 (a) and Fig. 7 (b) along with the experimental results shown in Fig. 8. Feature extraction using SIFT points are represented by Fig. 9 (a) and Fig. 9 (b). Therefore, for the creation of catalogue at least 30 foliage models were contemplated and planned using each methodology.

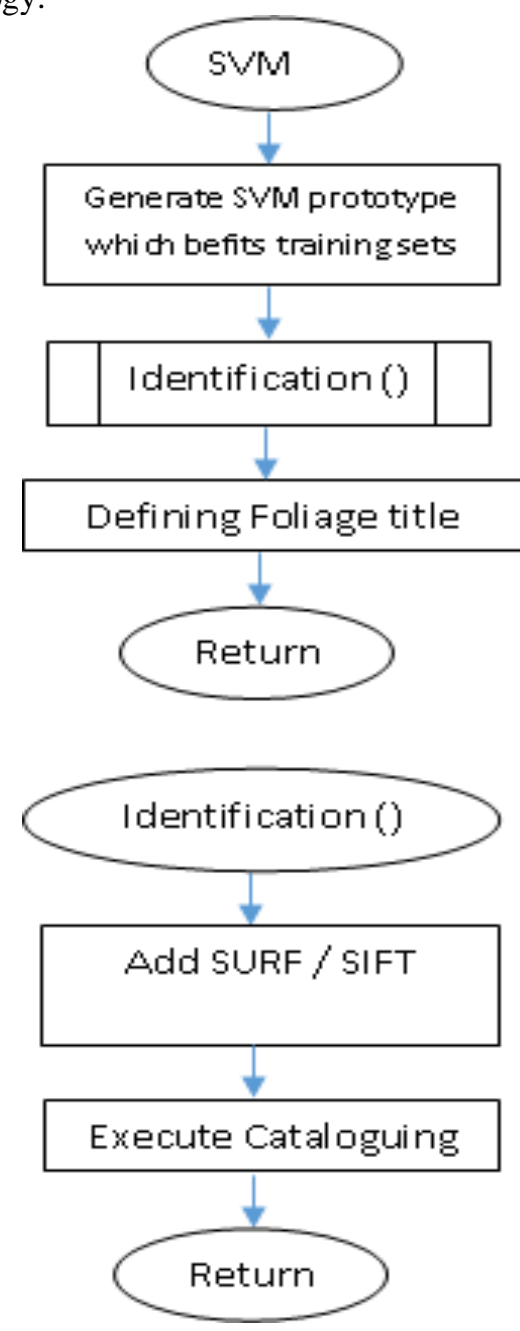

Fig 6. SVM Flow Diagram

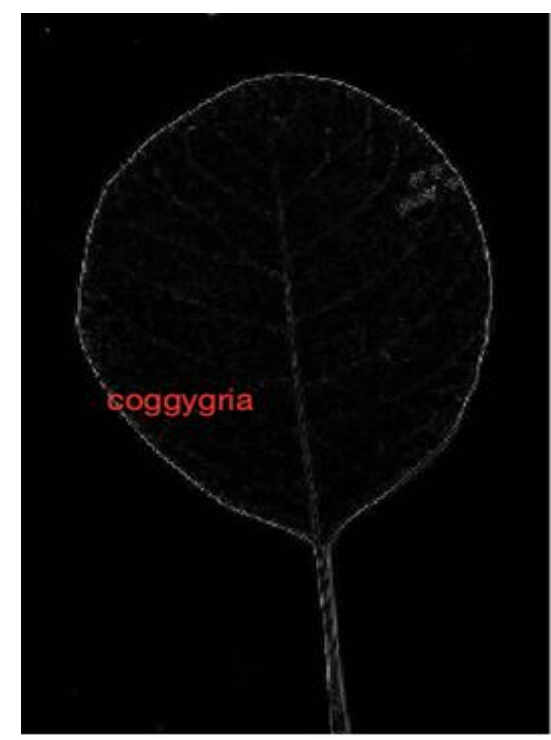

Fig. 7 (a). Leaf Identification using SURF

SVM requires a novel set of input pictograph which would undergo the similar procedure after which the input matrix from SURF and SIFT would then be equated with the matrix which was stored in the catalogue. Then SVM would categorize that contains the maximum notch, and the aforementioned would be exhibited.

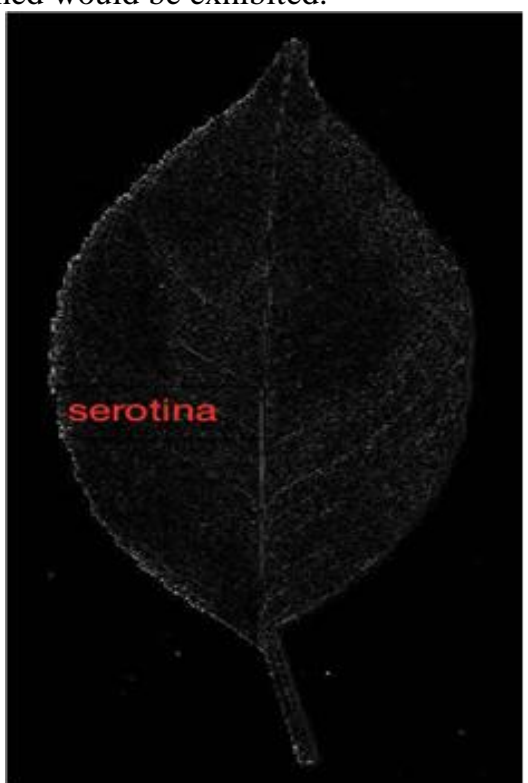

Fig. 7 (b). Leaf Identification using SURF

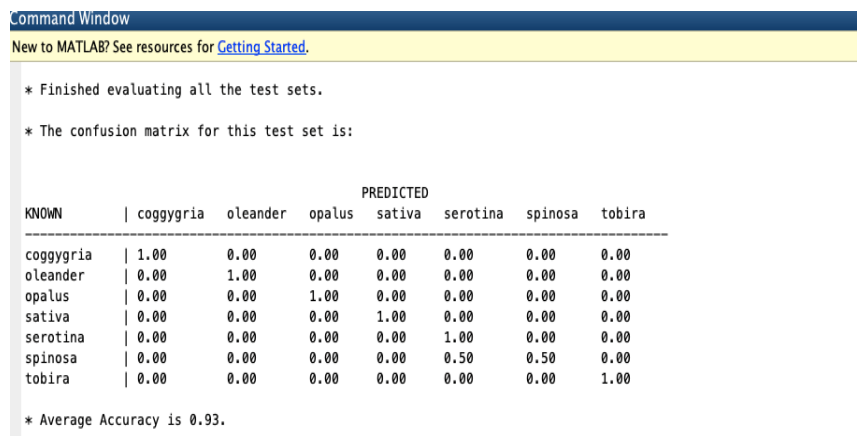

Fig 8. Experimental Results Using SURF

The

methodologies

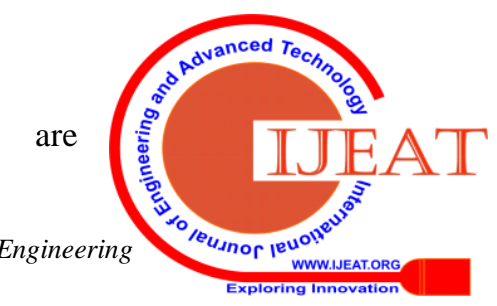


implemented. The leaf images are normalised because of the dimension and location of the data file. To evaluate the performances of each method a recognition model will be generated by means of an array of values meant for individual herbal species.

The confusion matrix displayed in Table 1 represents the outcomes of the assessment done with the help of SURF algorithm. Hence leaves are codified by the alphabets from A to $G$ aimed at documentation. Each plant leaf was tested 10 times.

The confusion matrix displayed in Table 2 represents the outcomes of the assessment done with the help of SIFT algorithm. Hence leaves are codified by the alphabets from A to $G$ aimed at documentation. Each plant leaf was tested 10 times.

Based on table 1, the accuracy (ACC) is calculated with the help of Equation 1 whereas the error rate (ERR) is generated through Equation 2 as mentioned hereunder:

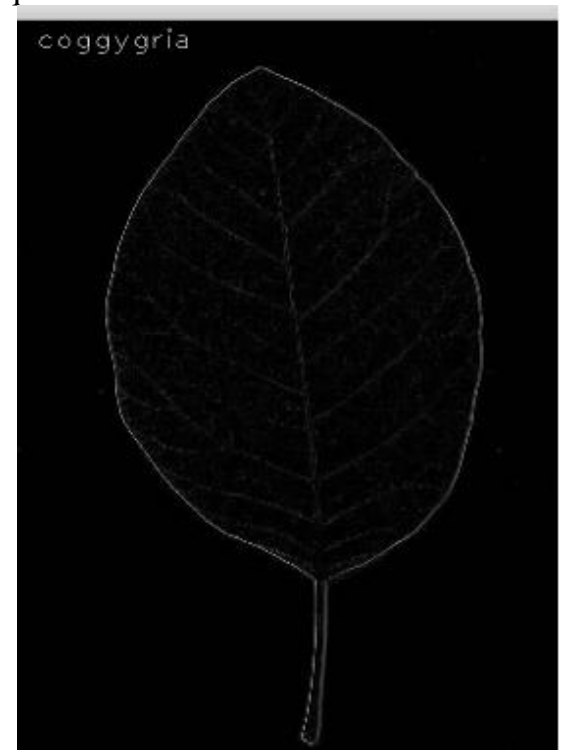

Fig. 9 (a). Leaf Identification using SIFT

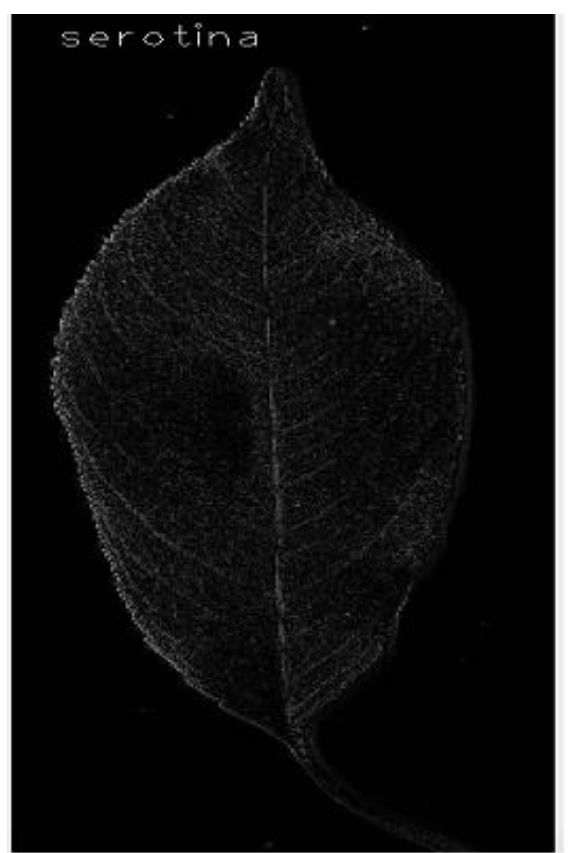

Fig. 9 (b). Leaf Identification using SIFT

$$
\begin{aligned}
& A C C=\left[\frac{T P+T N}{T P+T N+F N+F P}\right] * 100 \\
& E R R=\left[\frac{F P+F N}{T P+T N+F N+F P}\right] * 100
\end{aligned}
$$

Resulting Equations 1 and 2, the ACC of the system for perfectly recognizing herbal foliage using SURF was 93\% whereas the ERR of the system erroneously recognizing was $7 \%$.

From table 2, the accuracy (ACC) is calculated utilizing Equation 1 was $78 \%$ whereas the error rate (ERR) was $22 \%$ identified through Equation 2.

Table- I: Confusion Matrix for SURF

\begin{tabular}{|l|l|l|l|l|l|l|l|l|}
\hline \multicolumn{1}{c|}{} & \multicolumn{2}{|c|}{ PREDICTED } \\
\cline { 2 - 9 } & & $\mathrm{A}$ & $\mathrm{B}$ & $\mathrm{C}$ & $\mathrm{D}$ & $\mathrm{E}$ & $\mathrm{F}$ & $\mathrm{G}$ \\
\cline { 2 - 9 } & $\mathrm{A}$ & 10 & 0 & 0 & 0 & 0 & 0 & 0 \\
\cline { 2 - 9 } & $\mathrm{B}$ & 0 & 10 & 0 & 0 & 0 & 0 & 0 \\
\cline { 2 - 9 } & $\mathrm{C}$ & 0 & 0 & 10 & 0 & 0 & 0 & 0 \\
\cline { 2 - 9 } & $\mathrm{D}$ & 0 & 0 & 0 & 10 & 0 & 0 & 0 \\
\cline { 2 - 9 } & $\mathrm{E}$ & 0 & 0 & 0 & 0 & 10 & 0 & 0 \\
\cline { 2 - 9 } & $\mathrm{F}$ & 0 & 0 & 0 & 0 & 5 & 5 & 0 \\
\cline { 2 - 9 } & $\mathrm{G}$ & 0 & 0 & 0 & 0 & 0 & 0 & 10 \\
\hline
\end{tabular}

Table- II: Confusion Matrix for SIFT

\begin{tabular}{|l|l|l|l|l|l|l|l|l|}
\hline \multicolumn{1}{|c|}{} & \multicolumn{6}{|l|}{ PREDICTED } \\
\cline { 2 - 9 } & & $\mathrm{A}$ & $\mathrm{B}$ & $\mathrm{C}$ & $\mathrm{D}$ & $\mathrm{E}$ & $\mathrm{F}$ & $\mathrm{G}$ \\
\cline { 2 - 9 } & $\mathrm{A}$ & 4 & 0 & 0 & 3 & 0 & 0 & 3 \\
\cline { 2 - 9 } & $\mathrm{B}$ & 0 & 9 & 0 & 0 & 0 & 0 & 1 \\
\cline { 2 - 9 } & $\mathrm{C}$ & 0 & 0 & 10 & 0 & 0 & 0 & 0 \\
\cline { 2 - 9 } & $\mathrm{D}$ & 0 & 0 & 0 & 9 & 0 & 1 & 0 \\
\cline { 2 - 9 } & $\mathrm{E}$ & 0 & 0 & 0 & 0 & 10 & 0 & 0 \\
\cline { 2 - 9 } & $\mathrm{F}$ & 0 & 0 & 0 & 0 & 5 & 5 & 0 \\
\cline { 2 - 9 } & $\mathrm{G}$ & 0 & 0 & 0 & 0 & 0 & 0 & 10 \\
\hline
\end{tabular}

\section{CONCLUSION}

The objective of this research paper is to find the most efficient method for recognizing medicinal plants correctly. By using the Speeded Up Robust Feature (SURF) algorithm different medicinal leaves are recognized 93\% accurately compared to Scale Invariant Feature Transform (SIFT) algorithm. Based on the analysis, it is confirmed that the recognition rate of SURF is better than SIFT. In this direction the accuracy can be increased to $100 \%$ by increasing the size of the trained data set.

\section{REFERENCES}

1. E. Sandeep Kumar, "Leaf colour, area and edge features-based approach for identification of Indian medicinal plants", Indian Journal of Computer Science and Engineering, Vol.3, No.3, pp. 436 442, 2012.

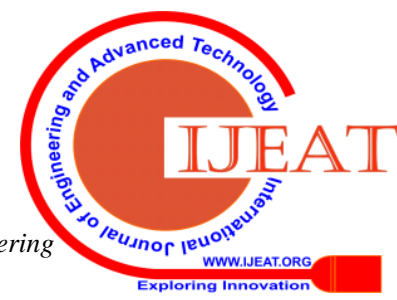


2. C. Ananthi, Azha. Periasamy, S. Muruganand, "Pattern recognition of medicinal leaves using image processing techniques", Journal of Nano Science and Nanotechnology, Vol.2, Issue 2, pp. 214 - 218 , 2014

3. S. Jesse Dave Selda, R. Roi Martin Ellera, C. Leandro Cajayon II, B. Noel Linsangan, "Plant identification by image processing of leaf veins", Proc. of the International Conference on Imaging, Signal Processing and Communication, pp. 40 - 44, 2017.

4. T. Vijayashree, A. Gopal, "Authentication of leaf image using image processing technique", ARPN Journal of Engineering and Applied Sciences, Vol.10, No.9, pp. 4287 - 4291, 2015.

5. D. Sachin Chothe, V.R. Ratnaparkhe, "Plant identification using leaf Images", International Journal of Innovative Research in Science, Engineering and Technology, Vol.4, Special Issue 6, pp. 659 - 664 2015.

6. B.R. Pushpa, C. Anand, P. Mithun Nambiar, "Ayurvedic plant species recognition using statistical parameters on leaf Images", International Journal of Applied Engineering Research, Vol.11, No.7, pp. $5142-5147,2016$.

7. S. James Cope, David Corney, Y. Jonathan Clerk, Paolo Remagnino, Paul Wilkin, "Plant species identification using digital morphometrics: A review", An International Journal of Expert Systems with Applications, Vol.39, No.8, pp. 7562 - 7573, 2012.

8. K. Pankaja, G. Thippeswamy, "Survey on leaf recognition and classification", Proc. of the International Conference on Innovative Mechanisms for Industry Applications, pp. 442 - 450, 2017.

9. 9.A. Akshay Patil, K. S. Bhagat, "Plants identification by leaf shape recognition: A review", International Journal of Engineering Trends and Technology, Vol.35, No.8, pp. 359 - 361, 2016.

10. 10. L. Kue-Bum, H. Kwang-Seok, "An Implementation of leaf recognition system using leaf vein and shape", International Journal of Bio-Science and Bio-Technology, Vol.5, No.2, pp. 57 - 66, 2013.

\section{AUTHORS PROFILE}

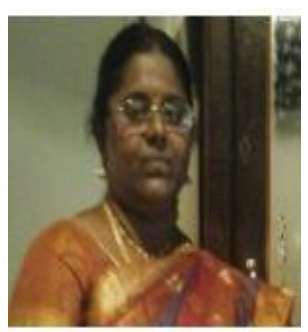

PL. Chithra is a Professor in the Department of Computer Science at the University of Madras, Chennai, Tamil Nadu, India. She received her M.C.A and Ph.D. degrees from Alagappa University and University of Madras, Tamil Nadu, India. She has been serving as an Organizing Chair and Program Chair for several International conferences. She has been supervising Ph.D. and M.Phil research scholars. Her area of specialization includes Image Processing Techniques, Big data analytics and Network Security. She has published more than 60 research papers in national and international journals.

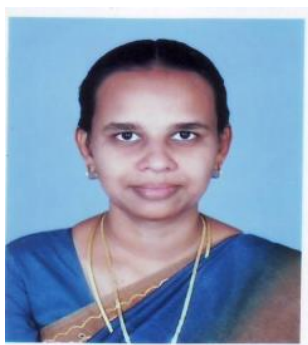

S. Janes Pushparani is an Assistant Professor in the department of Bachelor of Computer Applications, Ethiraj College for Women, Chennai, Tami Nadu, India. She had received her Bachelor of Physics, Master of Computer Applications and Master of Philosophy in Computer Science from Bharathidasan University, Tiruchirappalli, India. She is currently pursuing her doctoral degree at University of Madras and currently working on Digital Image Processing area. 